\title{
Contribuição dos parâmetros biomecânicos para o desempenho de saltos verticais de jogadoras de basquetebol
}

CDD. 20.ed. 796.023

796.32

\author{
Rodrigo Maciel ANDRADE* \\ Alberto Carlos AMADIO* \\ Júlio Cerca SERRÃO* \\ Maria Augusta Peduti Dal'Molin KISS* \\ Alexandre MOREIRA*
}

\begin{abstract}
Resumo
0 objetivo do presente estudo foi investigar a contribuição dos parâmetros biomecânicos para 0 desempenho do salto vertical com contramovimento (SV) e SV precedido de corrida (SV corrida $_{\text {) }}$ em 19 jogadoras da seleção brasileira adulta de basquetebol feminino $(26,2 \pm 4,7$ anos; $1,81 \pm 0,07 \mathrm{~m} ; 75,6$ $\pm 12,6 \mathrm{~kg} ; 20,4 \pm 6,0 \%$ de gordura). Foram considerados os picos de força passiva (PFPa) e propulsão (PFP), tempo para alcance dos picos de força passiva (TPFPa) e propulsão (TPFP), "load rate" (LR), taxa de desenvolvimento de força (TDF), tempo de fase excêntrica $\left(T_{\text {exc }}\right)$ e concêntrica $\left(T_{\text {con }}\right)$. A análise de componentes principais revelou que 50,86\% da altura de SV foi explicada por PFPa, TPFPa, LR, $T_{\text {exc }}$ e TPFP,

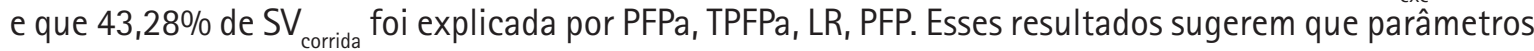
temporais parecem contribuir de maneira mais significativa para o desempenho de salto, porem diferentes tipos de salto podem demandar comportamentos distintos de parâmetros biomecânicos.
\end{abstract}

UNITERMOS: Análise de componentes principais; Performance; Biomecânica; Força de reação do solo.

\section{Introdução}

A utilização de testes de saltos verticais com o objetivo de monitorar ou avaliar as respostas do treinamento no basquetebol é bastante difundida na literatura (ZIV \& LiDOR, 2009), tendo como base de sustentação, a peculiaridade quanto as exigências do jogo, como por exemplo, as açôes de "bloqueios", "rebotes", "passes" e "arremessos" (BEN ABDELKRIM, El FazAa \& El ATı, 2007). Sendo assim, é crescente o número de investigações que têm sido conduzidas levando em consideração as variáveis atreladas ao desempenho nessas açôes e fundamentos do jogo.

Alguns estudos apontam que o desempenho no salto vertical, portanto, a altura obtida no salto vertical, depende fundamentalmente da geração da força de forma mais breve possível. DE RUITER, VAN Leeuwen, Heijblom, Bobbert e De HaAn (2006), por exemplo, encontraram coeficientes de correlação de 0,76 a 0,86 entre a altura de salto vertical e o torque gerado a 40 milésimos de segundo, corroborando com os resultados de Thorlund, AagaArd e MadSEN
(2009), que verificaram coeficiente de correlação de $0,74(\mathrm{p}<0,05)$ entre a taxa de desenvolvimento de força gerada a 200 milésimos de segundo para extensores de joelho e a altura de salto vertical, e com Thorlund, Michalsik, Madsen e Aagaard (2008), que reportaram diminuição aguda da taxa de desenvolvimento de força e da altura de salto vertical. Adicionalmente, outros estudos da literatura também demonstraram o efeito substancial do incremento na taxa de desenvolvimento de força na altura de salto vertical, após um período de treinamento (Kyrolainen, Avela, McBride, Koskinen, Andersen, Sipila, Takala \& Komi, 2005).

Por outro lado, estudos também refutam tais resultados e apontam uma maior contribuição para a altura do salto vertical a partir de parâmetros como o pico de força produzida na tarefa (ANDRADE, 2009; Kollias, Hatzitaki, Papaiakovou \& Giatsis, 2001; LafFaye, Bardy \& Durey, 2007). Nesse sentido, Nuzzo, McBride, Cormie e McCaulley (2008) 
encontraram correlação significante $(r=0,69)$ entre força máxima relativa à massa corporal e altura de salto vertical. Do mesmo modo, KaWAMORI, RossI, Justice, Haff, Pistilli, O’Bryant, Stone e Haff (2006) demonstraram que o pico de força apresentou maior magnitude de correlação com a altura de salto vertical $(r=0,82)$ do que a taxa de desenvolvimento de força $(r=0,14)$. Resultado semelhante ao descrito por Ugarkovic, Matavulj, KuKolj e Jaric (2002), que reportaram coeficientes de correlações de $\mathrm{r}=0,38$ a r $=0,52$ entre força máxima e altura de salto vertical, e de $r=0,02$ a $r=0,03$ entre taxa de desenvolvimento de força e altura de salto vertical, e de Ugrinowitsch, Tricoli, Rodacki, Batista e RICARD (2007) que encontraram que a força máxima parece ser mais importante para a obtenção de uma maior altura do salto vertical do que a taxa de desenvolvimento de força.

Sendo assim, pode-se perceber que ainda se está longe de um consenso acerca da contribuição dos parâmetros biomecânicos atrelados a força muscular para o desempenho (altura) no salto vertical. Ainda, diante da ampla utilização de testes de salto vertical em diferentes dimensões, seja no monitoramento de um processo de treinamento, ou mesmo na busca de associação entre esse indicador de força explosiva com outras ações e testes de desempenho (por exemplo, com teste de aceleração e velocidade), parece ser imperativo a busca por maior entendimento quanto as variáveis determinantes de desempenho de salto vertical.

Ainda que seja considerado o relevante papel dos aspectos coordenativos no desempenho do salto vertical (RODACKI, FOWLER \& BENNETT, 2001; UGRINOWITSCH et al., 2007) é razoável admitir que a força muscular, em suas distintas manifestações, como força máxima, força pico e taxa de desenvolvimento de força, contribuam de forma importante para o incremento da capacidade de salto vertical, entretanto, é fundamental que se avance no conhecimento da contribuição relativa dessas manifestaçôes.

Outra questão a ser considerada é que a grande maioria dos estudos centrados nas análises do desempenho de salto vertical e das contribuições de diferentes manifestaçōes de força e parâmetros biomecânicos, o fizeram a partir de análises de correlação bivariada. Embora essa análise seja de grande valia para o entendimento da associação entre o desempenho no salto vertical e os parâmetros biomecânicos, tem sido apontado que essa abordagem pode ter limitações quanto ao entendimento da relação entre variáveis dependentes e independentes, em fenômenos complexos e de elevada redundância (Kollias et al., 2001; Laffaye, Bardy \& Durey, 2007), como podem ser considerados os movimentos de salto vertical (RODACKI, Fowler \& BENNETT, 2001; UGRINOWITSCH et al., 2007).

Neste sentido, observa-se a tendência da adoção de análises multivariadas, como a análise de componentes principais. Com esta análise, alguns estudos têm revelado que o desempenho no salto vertical apresenta maior variação explicada por parâmetros predominantemente temporais (ANDRADE, 2009; Kollias et al., 2001; Laffaye, Bardy \& Durey, 2007), o que sinaliza para um avanço no conhecimento acerca do desempenho no salto vertical. Todavia, tais contribuiçôes também indicam que a contribuição desses parâmetros talvez seja amostradependente e teste-dependente (Kollias et al., 2001; Laffaye, Bardy \& Durey, 2007; Meylan, McMaster, Cronin, Mohammad, Rogers \& Deklerk, 2009; Smirniotou, Katsikas, Paradisis, Argeitaki, Zacharogiannis \& Tziortzis, 2008).

Assim, o objetivo deste estudo foi investigar os parâmetros biomecânicos atrelados à força muscular (como força pico, tempo para força pico, taxa de desenvolvimento de força, dentre outros) de maior contribuição para o desempenho em dois tipos de salto vertical (salto vertical com contramovimento e o salto vertical com contramovimento precedido de corrida de aproximação) em jogadoras de basquetebol de alto rendimento, partindo-se da premissa de que por além destes tipos de saltos serem frequentemente realizados durante uma partida de basquetebol, os saltos verticais são constantemente adotados como estratégias de avaliação e prescrição de treinamento, justificando a busca pelo melhor entendimento destas ações nesta população, uma vez que a contribuição dos parâmetros biomecânicos para a obtenção da maior altura possível parece ser amostra-dependente e teste-dependente (KolLIAS et al., 2001; Laffaye, Bardy \& Durey, 2007; MeYlan et al., 2009; SMirniotou et al., 2008; UGRINOWITSCH et al., 2007). 


\section{Materiais e métodos}

\section{Amostra}

A amostra foi selecionada por conveniência e contou com 19 jogadoras de basquetebol pertencentes à seleção brasileira feminina adulta que ocupava a quarta posição no "ranking" mundial no momento da investigação $(26,2 \pm 4,7$ anos; $1,81 \pm 0,07 \mathrm{~m} ; 75,6 \pm$ $12,6 \mathrm{~kg} ; 20,4 \pm 6,0 \%$ gordura). Entre as jogadoras investigadas, havia medalhistas e participantes de Jogos Olímpicos, campeonatos mundiais, pan-americanos e sul-americanos. No momento de realização do estudo, a seleção se encontrava em período de preparação para a Copa América de 2009, competição na qual conquistou o primeiro lugar. Todas as participantes foram previamente orientadas sobre os objetivos do estudo e assinaram um termo de consentimento livre e esclarecido. Todos os procedimentos foram previamente aprovados pelo Comitê de Ética em Pesquisa da Escola de Educação Física e Esporte da Universidade de São Paulo sob o processo n. 2009/35.

\section{Procedimentos}

Para a realização do presente estudo, o departamento médico da seleção brasileira de basquetebol entrevistou cada atleta para o conhecimento do histórico clínico e condição ósteomioarticular do aparelho locomotor, para a identificação de possíveis alterações que pudessem interferir na realização dos testes de salto vertical. Nenhuma das atletas apresentou qualquer impedimento.

Após a realização dessa entrevista, e para caracterização da amostra, foi mensurado das voluntárias atletas às medidas antropométricas, que compreenderam a estatura e massa corporal, por meio de um estadiômetro (estadiômetro standart Sanny ${ }^{\circledR}$ - Brasil) e uma balança digital (Filizola ${ }^{\circledR}$ ID 1500 - Brasil), respectivamente. $\mathrm{O}$ percentual de gordura corporal (\% de gordura) foi estimado com o auxílio de um plicômetro Harpenden (Harpenden ${ }^{\circledR}$ - United Kingdom) para a mensuração das dobras cutâneas trícipital, subescapular e abdominal, além de fita métrica inextensível para determinação dos perímetros de coxa e perna (JaCKSON, POLlOCK \& WARD, 1980). A coleta dos dados foi realizada de acordo com a padronização proposta pela International Society for Advance of Kinanthropometry (NoRTON, WhitTINGHAM, CARTer, Kerr, Gore \& Marfell-Jones, 2005).

Após a realização das medidas antropométricas, as voluntárias foram submetidas aos testes de salto vertical, a fim de se identificar os parâmetros biomecânicos de maior contribuição para o desempenho dos mesmos. As voluntárias foram submetidas a um protocolo de aquecimento de 15 minutos, envolvendo exercícios de alongamento dinâmico e deslocamentos de baixa intensidade.

A aplicação dos testes e coleta de dados foi realizada em uma única sessão e em ordem randômica a fim de reduzir possíveis interferências da ordem de execução.

\section{Equipamentos}

\section{Plataforma de força}

Os parâmetros relacionados à força de reação do solo (FRS) foram mensurados por intermédio de uma plataforma de força dotada de sensores piezelétricos (Kistler AG, 9287 A).

Optou-se pela análise dos parâmetros biomecânicos obtidos apenas no vetor vertical de FRS, pois embora pelo preceito físico de lançamentos de projéteis, o deslocamento em parábola dependa de um vetor resultante produzido pelos vetores vertical e horizontal (Chaves \& Sampaio, 2007), já se tem conhecimento de que no teste de salto vertical, 97,3\% de todo trabalho corresponde ao vetor força vertical (HATZE, 1998).

\section{Teste de salto vertical}

Para a identificação dos parâmetros biomecânicos de maior contribuição para o desempenho nos testes de salto vertical, foram considerados dois tipos de salto vertical: o salto vertical com contramovimento (SV) e o salto vertical com contramovimento precedido de corrida de aproximação $\left(\mathrm{SV}_{\text {corrida }}\right)$.

\section{Salto vertical (SV)}

Como estratégia para a determinação de salto vertical, foi utilizado o teste de salto vertical com contramovimento e livre movimentação dos braços (SV), amplamente utilizado na literatura (BOBBERT \& CASIUS, 2005; BobBert, Gerritsen, LitJens \& VAn SOest, 1996; Bosco, Belli, Astrua, Tihanyi, Pozzo, Kellis, Tsarpela, Foti, Manno \& Tranquilli, 1995; Linthorne, 2001) e originalmente proposto por JOHNSON e NELSON (1974). Neste, não foi permitido saltitar ou realizar qualquer tipo de deslocamento antes da realização do salto, além do contramovimento. Foram realizadas três tentativas, com intervalo de um minuto entre elas. 
Salto vertical precedido de corrida de aproximação ( $\mathrm{SV}_{\text {corrida }}$ )

Com o propósito de atribuir maior validade ecológica ao teste de salto vertical, portanto, na tentativa de aproximar o teste de salto vertical ao experimentado por atletas de basquetebol durante as partidas, foi incluído um deslocamento em alta velocidade precedente a realização do salto vertical. Para tanto, foi inicialmente determinada e sinalizada no solo a distância de dois metros a partir da plataforma de força, distância próxima ao adotado na literatura (Fedie, Carlstedt, Willson \& KerNOZEK, 2010), sendo esta distância a ser percorrida no menor tempo possível. Para a mensuração do tempo para percorrer tal distância um sensor óptico foi posicionado em cada extremidade da distância de dois metros a ser percorrida.

Cada voluntária foi posicionada imediatamente atrás da linha demarcada no solo, onde após receber o sinal sonoro, atingiu a máxima velocidade possível para a distância e, imediatamente após ter cumprido a extensão de dois metros, e sobre a plataforma de força, realizou um salto buscando a máxima altura possível, com livre movimentação dos braços. A fase de propulsão bipodal e a fase de aterrissagem foram obrigatoriamente realizadas sobre a plataforma de força. A tentativa seria repetida caso ocorresse apoio unipodal, ou toque parcial do ante-pé e/ou retro-pé na plataforma, todavia, dado a familiarização com o teste, nenhuma atleta necessitou realizar nova tentativa. Foram realizadas três tentativas, com intervalo de um minuto entre elas, sendo a média retida para a análise.

\section{Identificação das fases de salto}

Embora anteriormente não tenha sido encontrada diferença significante $(\mathrm{p}<0,05)$ quanto a questões temporais relacionadas à fase excêntrica em diferentes tipos de salto vertical (UGRINOWITSCH et al., 2007), optou-se por considerar por além da fase concêntrica, também os parâmetros biomecânicos que emergiram da fase excêntrica, dado que, por se tratar de movimentos envolvendo o ciclo alongamento-encurtamento, é importante considerar a contribuição da fase excêntrica para o desempenho de salto (RODACKI \& FOWLER, 2001; RODACKI, FOWLER \& BENNETT, 2001).

Para a determinação dos parâmetros biomecânicos de SV foi considerado para início da fase de excêntrica $\left(t_{1}\right)$ o instante em que o vetor vertical de FRS apresentou redução de $5 \mathrm{~N}$ a partir da linha de base, conforme sugerido por García-López, Peleteiro, RodríguezMarroyo, Morante, Herrero e Villa (2005). Para a determinação da transição de fim de fase excêntrica e início da fase concêntrica $\left(t_{2}\right)$, inicialmente foi calculada a curva aceleração do centro de gravidade corporaltempo (aceleração do centro de gravidade corporal = FRS/massa corporal) do vetor vertical de FRS, e a partir desta, por dupla integração numérica calculada pela regra trapezoidal, foi obtida a curva posição do centro de gravidade corporal-tempo. Foi considerado o fim de fase excêntrica e início da fase concêntrica $\left(t_{2}\right)$ o instante de menor valor na curva posição do centro de gravidade corporal-tempo (LINTHORNE, 2001).

Como indicativo de fim da fase concêntrica $\left(\mathrm{t}_{3}\right)$ foi considerado o instante em que o vetor vertical de FRS apresentou valor igual a zero, conforme adotado em outros estudos (GARCía-LÓPEZ et al., 2005; LINTHORNE, 2001; UGRINOWITSCH et al., 2007) (FIGURA 1).

FRS = Força de reação

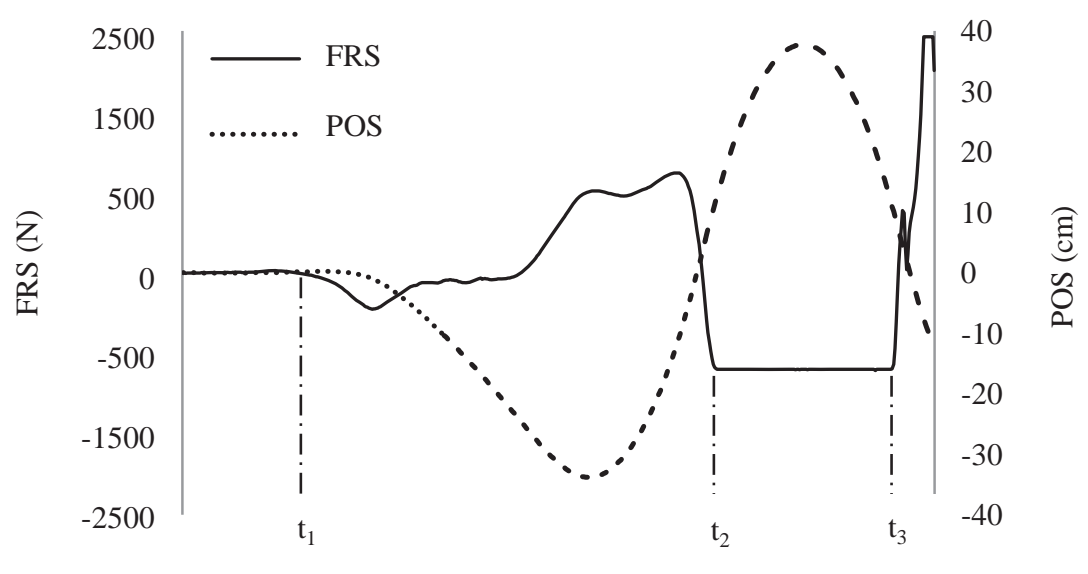

Tempo (s)

FIGURA 1- Determinação dos instantes de início e fim das fases excêntrica e concêntrica de SV. 
Para a determinação dos parâmetros biomecânicos de $S V_{\text {corrida }}$, foi considerado para início da fase excêntrica $\left(\mathrm{t}_{1}\right)$ o instante em que a curva FRS-tempo apresentou valor maior do que zero.

$\mathrm{O}$ instante de transição de fim de fase excêntrica e início da fase concêntrica $\left(t_{2}\right)$, foi identificado pelo vetor horizontal (ântero-posterior) de FRS, sendo este o instante em que o vetor horizontal (ântero-posterior)

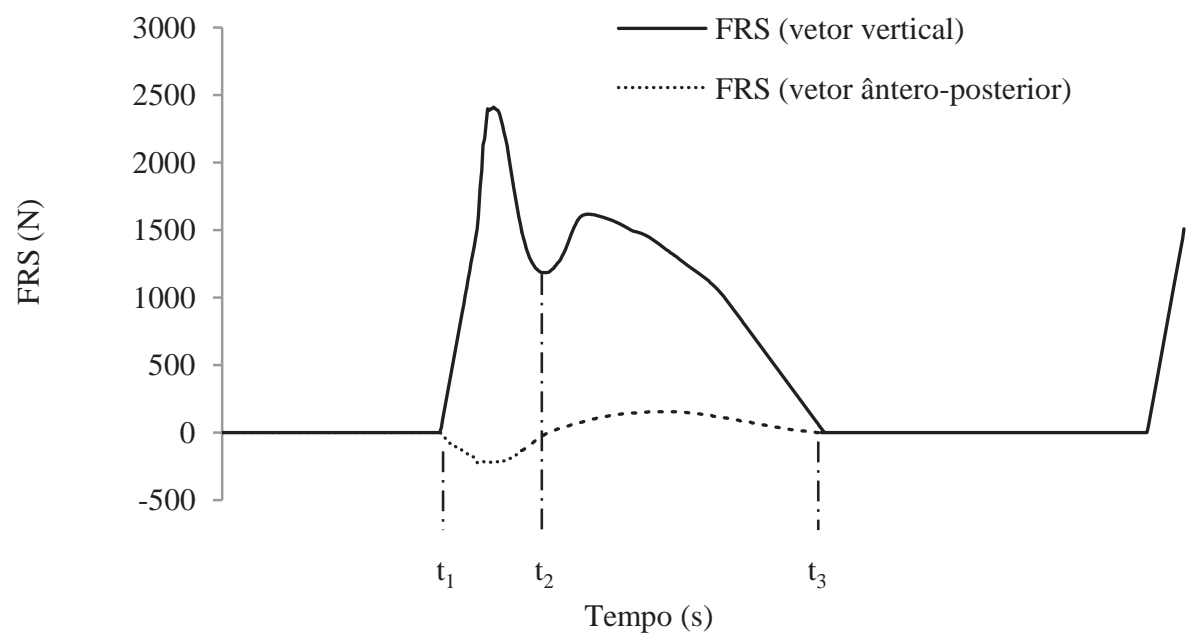

FIGURA 2 - Determinação dos instantes de início e fim das fases excêntrica e concêntrica de SV ${ }_{\text {corrida }}$.

\section{Parâmetros biomecânicos analisados}

Considerando que o impulso na fase concêntrica é determinante do desempenho no salto vertical (Komi \& Gollhofer, 1997), para investigar os parâmetros biomecânicos de maior contribuição para o desempenho, foram considerados os parâmetros que poderiam alterar a magnitude de geração do impulso, conforme adotado por UGRINOWITSCH et al. (2007). Sendo assim, e a exceção do parâmetro velocidade média de aproximação $\left(\mathrm{Vel}_{\text {média }}\right)$, obtido pela razão entre a distância percorrida de dois metros e o tempo para percorrer a mesma distância, e que foi calculada apenas para o teste $S V_{\text {corrida }}$, para ambos os testes de salto vertical (SV e SV $\mathrm{corrida}^{\text {a }}$ ).

Foram considerados os seguintes parâmetros biomecânicos (FIGURAS 3 e 4): Pico de força de passiva (PFPa): menor valor de FRS encontrado na fase excêntrica quando considerado SV e maior valor de FRS encontrado na fase excêntrica quando considerado $\mathrm{SV}_{\text {corrida }}$; Tempo para alcance do pico de força passiva (TPFPa): intervalo de tempo decorrido entre o início de fase excêntrica $\left(t_{1}\right)$ e PFPa; "Load rate" (LR): razão entre PFPa e TPFPa; Tempo de fase excêntrica $\left(T_{\text {exc }}\right)$ intervalo de tempo decorrido entre o início de fase excêntrica $\left(t_{1}\right)$ e início de fase concêntrica $\left(t_{2}\right)$; Pico de força de propulsão (PFP): maior valor de FRS de FRS apresentou valor positivo, como usualmente adotado em estudos de locomoção humana, como marcha, corrida e salto (CROss, 1999).

Como indicativo de fim da fase excêntrica $\left(t_{3}\right)$ foi considerado o instante em que o componente vertical de FRS apresentou valor igual à zero, sinalizando a perda de contato da voluntária atleta com o solo (plataforma de força) (FIGURA 2).

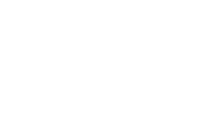
$\mathrm{N}=$ Newtons.

FRS $=$ Força de reação encontrado na fase concêntrica; Tempo para alcance do pico de força de propulsão (TPFP): intervalo de tempo decorrido entre o início de fase concêntrica $\left(t_{2}\right)$ e PFP; Taxa de desenvolvimento de força (TDF): razão entre PFP e TPFP. Tempo de fase concêntrica $\left(T_{c o n}\right)$ : intervalo de tempo decorrido entre início de fase concêntrica $\left(t_{2}\right)$ e fim de fase concêntrica $\left(t_{3}\right)$.

\section{Análise de dados}

\section{Tratamento matemático}

A FRS foi mensurada com frequência de amostragem de $1 \mathrm{kHz}$. Com a finalidade de minimizar o efeito de ruídos originários de fatores externos e internos, os dados provenientes da plataforma de força foram submetidos ao filtro Butterworth de $4^{a}$ ordem do tipo passa baixa, com frequência de corte de $40 \mathrm{~Hz}$, definida por análise residual (WINTER, 2004). As rotinas matemáticas utilizadas para o tratamento de dados foram desenvolvidas em ambiente MATLAB $^{\circledR}$ (versão R2006a).

A altura de salto, indicativa de despenho, foi determinada a partir do impulso, conforme sugerido pela literatura (BACA, 1999; LINTHORNE, 2001).

Para cada tentativa, foram obtidos os parâmetros biomecânicos e o cálculo da altura de salto. Todavia, 
foi retido para análise, a média dos valores obtidos nas três tentativas para cada um dos parâmetros biomecânicos analisados, assim como para a altura de salto.

\section{Tratamento estatístico}

Inicialmente recorreu-se ao teste de normalidade de Shapiro-Wilk e então se seguiu a apresentação da estatística descritiva (média e desvio-padrão). Posteriormente, adotou-se a técnica multivariada de análise de componentes principais, a fim de se observar a formação de componentes e as respectivas contribuiçōes dos parâmetros para cada componente formado, análise esta assegurada como técnica adequada, após a realização do teste de esfericidade de Bartlett. Determinou-se o número máximo de componentes sendo em seguida retidos somente aqueles com autovalor igual ou maior que 1 ; a partir da matriz original houve a rotação pelo método varimax (rotação ortogonal).

Todas as análises foram realizadas no pacote estatístico SPSS ${ }^{\circledR}$ versão 15.0 para Windows ${ }^{\circledR}$ (SPSS Inc, Chicago).
FIGURAS 3 e 4

FRS = Força de reação do solo;

$\mathrm{N}=$ Newtons

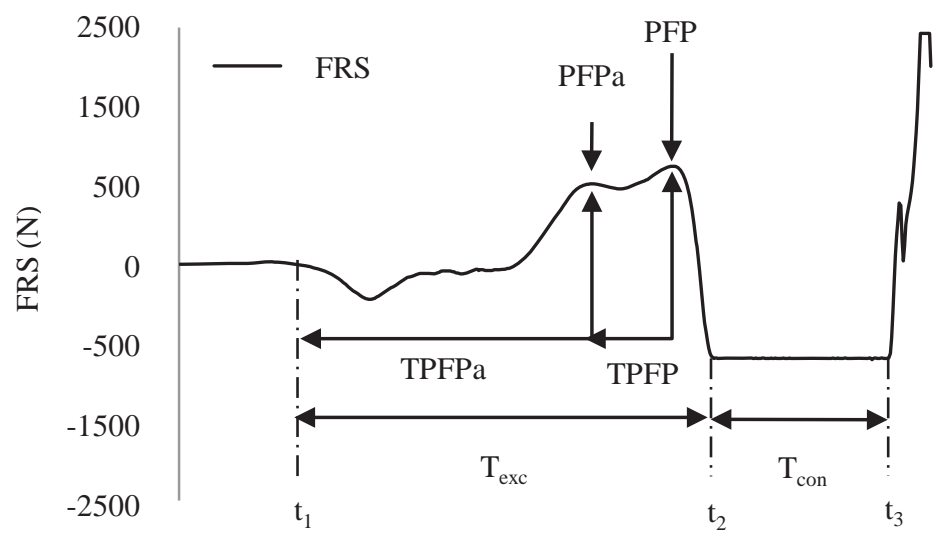

Tempo (s)

FIGURA 3 - Determinação dos parâmetros biomecânicos obtidos a partir de SV.

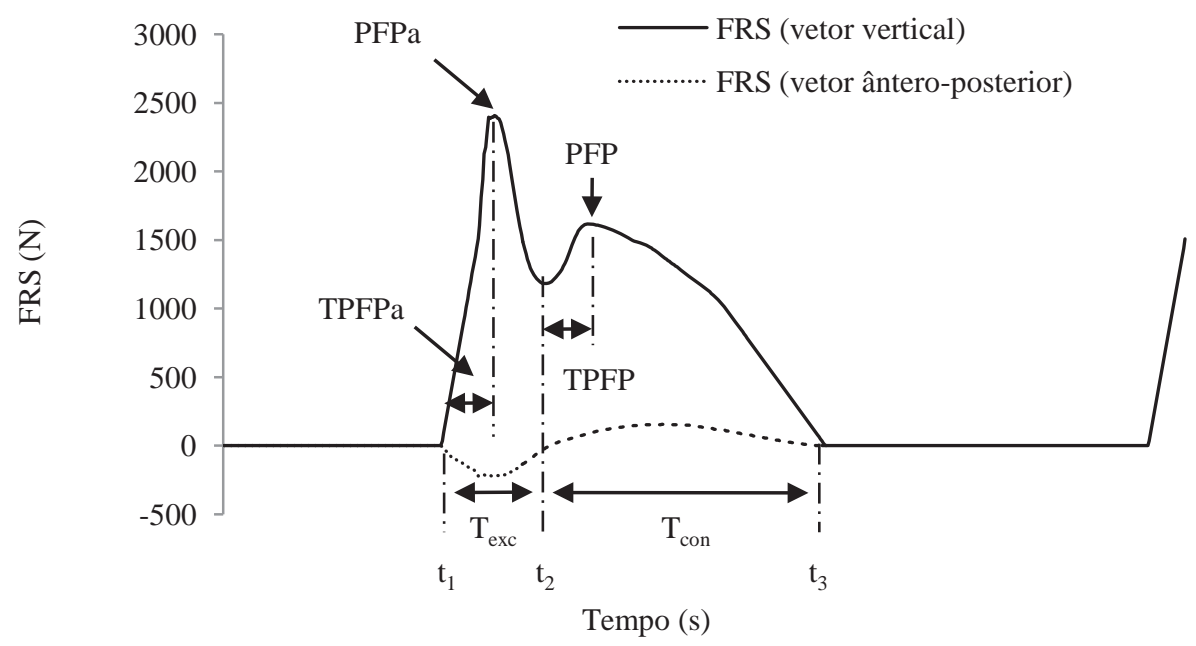

FIGURA 4 - Determinação dos parâmetros biomecânicos obtidos a partir de $\mathrm{SV}_{\text {corrida }}$. 


\section{Resultados}

Os valores obtidos para os parâmetros biomecânicos de $S V(h=0,29 \pm 0,02 \mathrm{~m})$ e SV $\mathrm{corrida}_{\text {a }}(\mathrm{h}=0,34$ $\pm 0,03 \mathrm{~m}$ ) são mostrados nas TABELA 1 .

A matriz de fatores gerados a partir de SV e de $\mathrm{SV}_{\text {corrida }}$ e os parâmetros incluídos em cada componente são apresentadas nas TABELA 2.

Para o SV foram retidos dois componentes. O componente 1 respondeu por $50,86 \%$ da variância total, com maior carga ocasionada pelos parâmetros PFPa, TPFPa, LR, $T_{\text {exc }}$ e TPFP. O componente 2 respondeu por uma variação de $22,53 \%$, sendo composto pelos parâmetros PFP, TDF e $\mathrm{T}_{\text {con }}$ (TABELA 2).

Ao considerar o teste $\mathrm{SV}_{\text {corrida }}$, três componentes foram retidos. O componente 1, respondeu por $43,28 \%$ da variância total, com maior carga ocasionada pelos parâmetros PFPa, TPFPa, LR, PFP. O componente 2 respondeu por uma variação de $20,03 \%$, sendo composto pelos parâmetros $\mathrm{T}_{\text {exc }}$, TPFP e TDF; O componente 3 respondeu $15,84 \%$ sendo formado pelos parâmetros $\mathrm{Vel}_{\text {média }}$ e $\mathrm{T}_{\text {exc }}$ (TABELA 2).

TABELA 1- Média e DP dos parâmetros biomecânicos obtidos no salto vertical (SV) e no salto vertical com contramovimento precedido de corrida de aproximação $\left(\mathrm{SV}_{\text {corrida }}\right)$.

\begin{tabular}{|c|c|c|c|c|c|}
\hline \multirow[b]{2}{*}{ Parâmetros } & \multicolumn{2}{|c|}{ SV } & \multicolumn{2}{|c|}{$\mathrm{SV}_{\text {corrida }}$} & \multirow{11}{*}{$\begin{array}{l}\text { TABELA } 1 \text { e } 2 \\
\left(\mathrm{~ms}^{-1}\right)=\text { milésimo de } \\
\text { segundo; } \\
(\mathrm{N})=\text { Newton; } \\
\text { (s) = segundo. }\end{array}$} \\
\hline & Média & DP & Média & DP & \\
\hline $\mathrm{Vel}_{\text {média }}\left(\mathrm{m} \bullet \mathrm{s}^{-1}\right)$ & - & - & 3,45 & 0,40 & \\
\hline $\operatorname{PFPa}(\mathrm{N})$ & $-384,73$ & 129,36 & 2470,91 & 676,37 & \\
\hline TPFPa (s) & 0,17 & 0,04 & 0,08 & 0,03 & \\
\hline $\mathrm{LR}\left(\mathrm{N} \cdot \mathrm{ms}^{-1}\right)$ & $-2,79$ & 1,24 & 43,81 & 23,44 & \\
\hline $\mathrm{T}_{\mathrm{exc}}(\mathrm{s})$ & 0,38 & 0,09 & 0,11 & 0,06 & \\
\hline $\operatorname{PFP}(\mathrm{N})$ & 1542,02 & 498,73 & 2629,93 & 510,05 & \\
\hline TPFP (s) & 0,13 & 0,08 & 0,02 & 0,01 & \\
\hline $\operatorname{TDF}\left(\mathrm{N} \cdot \mathrm{ms}^{-1}\right)$ & 26,26 & 21,14 & 205,30 & 130,02 & \\
\hline $\mathrm{T}_{\text {con }}(\mathrm{s})$ & 0,30 & 0,09 & 0,19 & 0,03 & \\
\hline
\end{tabular}

TABELA 2 - Variação percentual explicada para cada componente(VAR) ematriz de componentes do teste de salto vertical (SV) eno salto vertical com contramovimento precedido de corrida de aproximação (SV corrida $_{\text {) }}$.

\begin{tabular}{|c|c|c|c|c|c|}
\hline & \multicolumn{2}{|c|}{ SV } & \multicolumn{3}{|c|}{$S V_{\text {corrida }}$} \\
\hline & 1 & 2 & 1 & 2 & 3 \\
\hline VAR & 50,86 & 22,53 & 43,28 & 20,03 & 15,84 \\
\hline $\operatorname{Vel}_{\text {média }}\left(\mathrm{m} \bullet \mathrm{s}^{-1}\right)$ & - & - & 0,07 & 0,06 & 0,87 \\
\hline $\operatorname{PFPa}(\mathrm{N})$ & $-0,60$ & $-0,35$ & 0,94 & $-0,17$ & 0,01 \\
\hline TPFPa (s) & 0,93 & $-0,06$ & $-0,94$ & $-0,10$ & 0,04 \\
\hline $\mathrm{LR}\left(\mathrm{N} \cdot \mathrm{ms}^{-1}\right)$ & $-0,96$ & $-0,12$ & 0,96 & $-0,11$ & 0,17 \\
\hline $\mathrm{T}_{\mathrm{exc}}(\mathrm{s})$ & 0,84 & 0,39 & $-0,37$ & 0,69 & 0,14 \\
\hline $\operatorname{PFP}(\mathrm{N})$ & $-0,20$ & $-0,77$ & 0,82 & $-0,40$ & 0,17 \\
\hline TPFP $(s)$ & 0,93 & $-0,06$ & 0,11 & 0,85 & 0,22 \\
\hline $\mathrm{TDF}\left(\mathrm{N} \cdot \mathrm{ms}^{-1}\right)$ & 0,01 & $-0,74$ & 0,15 & $-0,73$ & 0,27 \\
\hline $\mathrm{T}_{\text {con }}(\mathrm{s})$ & 0,30 & 0,79 & $-0,05$ & $-0,01$ & $-0,83$ \\
\hline
\end{tabular}




\section{Discussão}

Os resultados da análise de componentes principais revelaram que o desempenho no salto vertical (SV) apresentou maior variação $(50,86 \%)$ explicada pelo componente 1, composto pelos parâmetros pico de força passiva, tempo para pico de força passiva, "load rate", tempo de fase excêntrica e tempo para pico de força de propulsão; enquanto que o salto vertical precedido de corrida $\left(\mathrm{SV}_{\text {corrida }}\right)$ apresentou maior variação (43,28\%; componente 1) explicada pelos parâmetros pico de força passiva, tempo para pico de força passiva, "load rate" e pico de força de propulsão.

No que concerne à contribuição dos parâmetros biomecânicos para o desempenho no salto vertical, os resultados do presente estudo corroboram com outros da literatura que reportaram correlaçóes significantes entre a altura de salto vertical e pico de força produzida durante o salto (DowLING \& VAMOS, 1993), ou ainda, com resultados de investigações que revelaram coeficientes de correlações significantes, de diferentes magnitudes, entre o desempenho no salto vertical e pico de força mensurada na execução de diferentes exercícios, como o "leg press" (YAMAUCHI $\&$ IsHII, 2007), o agachamento (KAWAMORI et al., 2006), extensão de joelho (UGARKOVIC et al., 2002) e extensão de quadril (UGARKOVIC et al., 2002).

Entretanto, é importante destacar que os resultados desta investigação indicam que os parâmetros temporais parecem contribuir de maneira mais substancial e, portanto, com um papel ainda mais relevante para o desempenho de salto de jogadoras de basquetebol de alto rendimento, corroborando com resultados de investigações prévias de Dowling e Vamos (1993) e Cormie, McBride e McCaulley (2009).

Interessantemente, os resultados aqui apresentados revelam que apesar de ter sido evidenciado similar comportamento em $\mathrm{SV} \mathrm{e} \mathrm{SV}$ corrida no que diz respeito a variação explicada pelos componentes 1 e 2 , a magnitude dessas contribuiçóes difere dos achados de outros estudos. Kollias et al. (2001) e LAFFAYE, BARDY e DUREY (2007), encontraram contribuições mais equilibradas de tais componentes (35,7\% vs $41,1 \%$ e $41,1 \%$ vs $37,2 \%$, respectivamente). No entanto, cabe salientar que nesses estudos, os autores avaliaram em conjunto atletas de distintas modalidades esportivas, o que pode ter contribuído para tais divergências, visto que em função da demanda peculiar de cada esporte, a contribuição dos parâmetros para o desempenho parece ser diferente (Kollias et al., 2001; LAFFAYE, BARDY \& DUREY, 2007).
Outro importante resultado da presente investigação, diz respeito à contribuição teste-dependente dos parâmetros biomecânicos. O fato dos parâmetros tempo de fase excêntrica, tempo para pico de força de propulsão e pico de força de propulsão serem incluídos em diferentes componentes, tendo o tempo de fase excêntrica, e o tempo para pico de força de propulsão incluídos no componente $1 \mathrm{em} \mathrm{SV}$ e no componente $2 \mathrm{em} \mathrm{SV}$ corrida , e o pico de força de propulsão incluído no componente 2 em SV e no componente $1 \mathrm{em} \mathrm{SV}$ corrida , demonstra que a contribuição está associada ao tipo de teste e, portanto, a interpretação dessas associaçóes deve ser realizada com cautela e, principalmente, levando em consideração o tipo de teste realizado. É possível que a utilização de outros testes, tanto verticais quanto horizontais, apresentem contribuiçóes diferentes dos parâmetros, quando comparadas às encontradas no presente estudo, conforme sugerido em investigaçôes anteriores (MEYLAn et al., 2009; SMIRniotou et al., 2008; Ziv \& Lidor, 2009).

Nesse sentido, em adição à consideração da possibilidade de se verificar contribuiçóes distintas dos parâmetros biomecânicos para outros tipos de saltos, o que sugere a necessidade da realização de diferentes tipos de saltos nessa população para o avanço do conhecimento nessa área, também se faz necessário ressaltar que jogadores de basquetebol realizam durante a atividade competitiva, saltos em circunstâncias muito diferentes do salto vertical "estacionário" utilizado no presente estudo e amplamente investigado nos estudos com o basquetebol (ZIv \& LidoR, 2009); a realização de outros tipos de saltos, tanto durante as partidas, quanto em condições experimentais, poderia, hipoteticamente, aumentar a contribuição do vetor horizontal de força. Assim, é razoável admitirmos que análises de performance (ex.: "time-montion") conduzidas em jogos oficiais, preferencialmente com atletas de nível internacional, tendo como questão central, a identificação e o rastreamento da frequência dos diferentes tipos de saltos e parâmetros biomecânicos envolvidos, possam adicionar evidencias empíricas para essas suposições, estendendo nosso conhecimento nessa dimensão, e avançando os horizontes de pesquisadores e treinadores no que concerna a validade ecológica da utilização de determinados saltos, em situação de testes e avaliação.

No entanto, apesar de reconhecermos que a ausência de outros tipos de saltos seja uma limitação do presente estudo, é importante destacar que, por 
outro lado, o mesmo contribui com informaçôes importantes acerca da contribuição de parâmetros biomecânicos para os saltos verticais, que por sua vez, são frequentemente utilizados por pesquisadores no sentido de avaliar e monitorar atletas de basquetebol. DeleXtrat e COHEN (2009), por exemplo, utilizaram dois tipos de saltos verticais "estacionários" (um bipodal e outro unipodal) em jogadoras de basquetebol da Inglaterra e demonstraram não haver diferenças significantes entre atletas de diferentes posiçôes, tendo como variável dependente a altura de salto. Adicionalmente, o salto vertical "estacionário", ainda tem sido o salto mais comumente empregado nos estudos científicos no basquetebol, e ainda utilizado para comparar o componente anaeróbio de acordo com as posições e funções de jogo (Delextrat \& Cohen, 2009; Hoare, 2000; Lamonte, McKinney, Quinn, Bainbridge \& Eisenman, 1999).

Uma das possíveis razões para essa diferente contribuição dos parâmetros biomecânicos, e, portanto, a importância de se investigar a contribuição dos parâmetros biomecânicos em outros tipos de saltos, pode de algum modo estar associada às particularidades da magnitude da duração do ciclo alongamentoencurtamento. Embora seja difícil a diferenciação e dissociação quanto às contribuições do ciclo alongamento-encurtamento lento e rápido na execução do movimento humano, usualmente tem sido assumido que o ciclo alongamento-encurtamento lento implica em uma maior amplitude de movimento das articulaçóes e, em consequência disto, um maior tempo de contato e tempo de fase concêntrica, quando comparado ao ciclo alongamento-encurtamento rápido; assim, no ciclo alongamento-encurtamento lento, o estímulo mecânico para a realização do gesto parece ser preponderante e determinante. Por outro lado, no ciclo alongamento-encurtamento rápido, ocorre uma menor variação angular, menor tempo de contato e de fase concêntrica, tornando possivelmente a reposta neural o mecanismo de maior contribuição para o desempenho (SMIRNioTou et al., 2008).

$\mathrm{Na}$ presente investigação, as diferentes magnitudes temporais encontradas entre o tempo de fase excêntrica $(0,11$ vs 0,38$)$ e o tempo de fase concêntrica $(0,19$ vs 0,30$)$ nos teste $S V_{\text {corrida }}$ e SV, respectivamente, sinalizam para possíveis discrepâncias quanto as modulaçôes do ciclo alongamento-encurtamento. Sendo assim, os resultados corroboram com a ideia de que diferentes contribuições quanto às respostas do ciclo alongamento-encurtamento possam ocorrer entre os saltos analisados.

Uma possível explicação para as diferentes magnitudes temporais pode emergir quando da análise do componente 3 de SV ${ }_{\text {corrida }}$, que explicou 15,84\%, e foi composto pelos parâmetros velocidade média de aproximação e tempo de fase concêntrica. Considerando que velocidade média de aproximação e o tempo de fase concêntrica foram inseridos em um mesmo componente, poder-se-ia especular que este componente possa ter influenciado a amplitude de movimento durante a fase propulsiva do salto, e consequentemente, os demais parâmetros relacionados ao desempenho nessa ação, haja vista que modulações quanto à amplitude de movimento já foram evidenciadas quando comparado um único salto vertical com contramovimento a saltos verticais sucessivos (KomI, 1984b) e ao salto horizontal (ECKERT, 1968), o que levaria a diferentes respostas (mecânicas e neurais) do ciclo alongamento-encurtamento (Komi, 1984a).

\section{Conclusão}

A partir dos resultados pode-se concluir que os parâmetros biomecânicos, pico de força passiva, tempo para pico de força passiva, "load rate" e tempo de fase excêntrica para o teste de salto vertical com contramovimento, e pico de força passiva, tempo para pico de força passiva, "load rate" e pico de força de propulsão para o teste de salto vertical com contramovimento precedido de corrida de aproximação, respondem pela maior variação nos saltos verticais investigados.

Outrossim, a contribuição dos parâmetros para o desempenho do salto parece ser teste-dependente, uma vez que os parâmetros tempo de fase excêntrica, tempo para alcance do pico de força de propulsão e o pico de força de propulsão apresentaram contribuições distintas para os dois tipos de salto realizados. 


\begin{abstract}
Contribution of biomechanical parameters to vertical jump performance in basketball players
\end{abstract}

The aim of the study was to investigate the contribution of biomechanics parameters to counter-movement jump (CMJ) and CMJ preceded by run (CMJ ${ }_{\text {run }}$ ) in nineteen female basketball players from Brazilian National Team ( $26.2 \pm 4.7$ years; $1.81 \pm 0.07 \mathrm{~m} ; 75.6 \pm 12.6 \mathrm{~kg} ; 20.4 \pm 6.0 \%$ body fat). The parameters analyzed were passive (PaPF) and propulsion peak force (PPF), time to passive (TPaPF) and propulsion peak force (TPPF), load rate (LR), rate of force development (RFD), eccentric $\left(T_{\text {exc }}\right)$ and concentric phase duration $\left(\mathrm{T}_{\text {exc }}\right.$ ). The principal component analysis showed that $50.86 \%$ of $\mathrm{CMJ}$ performance was explained by PaPF, TPaPF, LR, $T_{\text {exc }}$ and TPPF, and $43.28 \%$ of $\mathrm{CMJ}_{\text {run }}$ was explained by PaPF, TPaPF, LR and PPF. These findings suggest that temporal parameters appear to have greater contribution to jump performance. Moreover, different types of jumps appear to induce distinct behavior of biomechanics parameters.

UnITERMs: Principal component analysis; Performance; Biomechanics; Ground reaction force.

\title{
Resumen
}

Contribución de los parámetros biomecánicos para el desempeño en saltos verticales de jugadoras de baloncesto

El objetivo de este estudio fue investigar la contribución de parámetros biomecánicos para el rendimiento

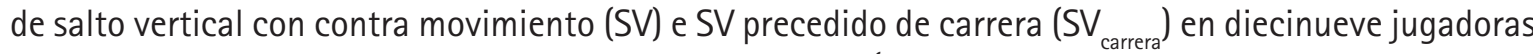
de baloncesto de la selección brasileña femenina adultas $(26,2 \pm 4,7$ años; $1,81 \pm 0,07 m ; 75,6 \pm 12,6$ $\mathrm{kg} ; 20,4 \pm 6,0 \%$ de grasa). Fueran considerados el pico de fuerza pasiva (PFPa) y propulsión (PFP), tiempo para llegar a picos de fuerza pasiva (TPFPa) y propulsión (TPFP), la tasa de carga (LR), tasa de desarrollo de fuerza (TDF), tiempo de la fase excéntrica $\left(T_{\text {exc }}\right)$ y concéntrica $\left(T_{\text {con }}\right)$. Las análisis de los componentes principales mostraron que $50,86 \%$ da la altura de SV fue explicada por PFPa, TPFPa, LR, $T_{\text {exc }}$ y TPFP, y que $43,28 \%$ de SV $V_{\text {carrera }}$ fue explicada por PFPa, TPFPa, LR, PFP. Los resultados sugieren parámetros temporales parecen contribuir más significativamente para rendimiento del salto pero, diferentes tipos del salto pueden demandar comportamientos distintos de los parámetros biomecánicos

PalabRas clave: Análisis de los componentes principales; Rendimiento; Biomecánica; Fuerza de reacción de suelo.

\section{Referências}

ANDRADE, R.M. Relação entre parâmetros biomecânicos e a aceleração no basquetebol. 2009. 78 f. Dissertação (Mestrado em Educação Física) - Escola de Educação Física e Esporte, Universidade de São Paulo, São Paulo, 2009.

BACA, A. A comparison of methods for analyzing drop jump performance. Medicine and Science in Sports and Exercise, Madison, v.31, n.3, p.437-42, 1999.

BEN ABDELKRIM, N.; EL FAZAA, S.; EL ATI, J. Time-motion analysis and physiological data of elite under-19-year-old basketball players during competition. British Journal of Sports Medicine, London, v.41, n.2, p.69-75, 2007.

BOBBERT, M.F.; CASIUS, L.J. Is the effect of a countermovement on jump height due to active state development? Medicine and Science in Sports and Exercise, Madison, v.37, n.3, p.440-6, 2005.

BOBBERT, M.F.; GERRITSEN, K.G.; LITJENS, M.C.; VAN SOEST, A.J. Why is countermovement jump height greater than squat jump height? Medicine and Science in Sports and Exercise, Madison, v.28, n.11, p.1402-12, 1996.

BOSCO, C.; BELLI, A.; ASTRUA, M.; TIHANYI, J.; POZZO, R.; KELLIS, S.; TSARPELA, O.; FOTI, C.; MANNO, R.; TRANQUILLI, C. A dynamometer for evaluation of dynamic muscle work. European Journal of Applied Physiology and Occupational Physiology, Berlin, v.70, n.5, p.379-86, 1995. 
CHAVES, A.; SAMPAIO, J.F. Movimento no plano e no espaço. In: Física básica: mecânica. Rio de Janeiro:

LTC, 2007. p.89-105.

CORMIE, P.; McBRIDE, J.M.; McCAULLEY, G.O. Power-time, force-time, and velocity-time curve analysis of the countermovement jump: impact of training. Journal of Strength and Conditioning Research, Champaign, v.23, n.1, p.177-86, 2009. CROSS, R. Standing, walking, running, and jumping on a force plate. American Journal of Physics, Lancaster, v.67, n.4, p.304-9, 1999.

DELEXTRAT, A.; COHEN, D. Strength, power, speed, and agility of women basketball players according to playing position. Journal of Strength and Conditioning Research, Champaign, v.23, n.7, p.1974-81, 2009.

DE RUITER, C.J.; VAN LEEUWEN, D.; HEIJBLOM, A.; BOBBERT, M.F.; DE HAAN, A. Fast unilateral isometric knee extension torque development and bilateral jump height. Medicine and Science in Sports and Exercise, Madison, v.38, n. 10, p.1843-52, 2006. DOWLING, J.J.; VAMOS, L. Identification of kinetic and temporal factors related to vertical jump performance. Journal of Applied Biomechanics, Champaign, v.9, p.95-110, 1993.

ECKERT, H.M. Angular velocity and range of motion in the vertical and standing broad jumps. Research Quarterly, Washington, v.39, n.4, p.937-42, 1968.

FEDIE, R.; CARLSTEDT, K.; WILLSON, J.D.; KERNOZEK, T.W. Effect of attending to a ball during a side-cut maneuver on lower extremity biomechanics in male and female athletes. Sports Biomechanics, Edinburgh, v.9, n.3, p.165-77, 2010. GARCÍA-LÓPEZ, J.; PELETEIRO, J.; RODRÍGUEZ-MARROYO, J.A.; MORANTE, J.C.; HERRERO, J.A.; VILLA, J.G. The validation of a new method that measures contact and flight times during vertical jump. International Journal of Sports Medicine, Stuttgart, v.26, p.294-302, 2005.

HATZE, H. Validity and reliability of methods for testing vertical jumping performance. Journal of Applied Biomechanics, Champaign, v.14, p.127-40, 1998.

HOARE, D.G. Predicting success in junior elite basketball players--the contribution of anthropometic and physiological attributes. Journal of Science and Medicine in Sport, Belconnen, v.3, n.4, p.391-405, 2000.

JACKSON, A.S.; POLLOCK, M.L.; WARD, A. Generalized equations for predicting body density of women. Medicine and Science in Sports and Exercise, Madison, v.12, n.3, p.175-81, 1980.

JOHNSON, B.L.; NELSON, J.K. The measurement of power. In:

Practical measurements for evaluation in physical education. Minnesota: Burgess, 1974. p.166-83.

KAWAMORI, N.; ROSSI, S.J.; JUSTICE, B.D.; HAFF, E.E.; PISTILLI, E.E.; O’BRYANT, H.S.; STONE, M.H.; HAFF, G.G. Peak force and rate of force development during isometric and dynamic mid-thigh clean pulls performed at various intensities. Journal of Strength and Conditioning Research, Champaign, v.20, n.3, p.483-91, 2006.

KOLLIAS, I.; HATZITAKI, V.; PAPAIAKOVOU, G.; GIATSIS, G. Using principal components analysis to identify individual differences in vertical jump performance. Research Quarterly for Exercise and Sport, Washington, v.72, n.1, p.63-7, 2001. KOMI, P.V. Biomechanics and neuromuscular performance. Medicine and Science in Sports and Exercise, Madison, v.16, n.1, p.26-8, 1984 .

. Physiological and biomechanical correlates of muscle function: effects of muscle structure and stretch-shortening cycle on force and speed. Exercise and Sport Sciences Reviews, Baltimore, v.12, n.1, p.81-121, $1984 \mathrm{~b}$.

KOMI, P.V.; GOLLHOFER, A. Stretch reflexes can have an important role in force enhancement during SSC exercise. Journal of Applied Biomechanics, Champaign, v.13, n.4, p.451-460, 1997.

KYROLAINEN, H.; AVELA, J.; McBRIDE, J.M.; KOSKINEN, S.; ANDERSEN, J.L.; SIPILA, S.; TAKALA, T.E.; KOMI, P.V. Effects of power training on muscle structure and neuromuscular performance. Scandinavian Journal of Medicine $\&$ Science in Sports, Copenhagen, v.15, n.1, p.58-64, 2005.

LAFFAYE, G.; BARDY, B.G.; DUREY, A. Principal component structure and sport-specific differences in the running one-leg vertical jump. International Journal of Sports Medicine, Stuttgart, v.28, n.5, p.420-5, 2007.

LAMONTE, M.; McKINNEY, J.; QUINN, S.; BAINBRIDGE, C.; EISENMAN, P. Comparison of physical and physiological variables for female college basketball players. Journal of Strength and Conditioning Research, Champaign, v.13, n.3, p.264-70, 1999. LINTHORNE, N.P. Analysis of standing vertical jumps using a force platform. American Journal of Physics, Lancaster, v.69, n.11, p.1198-1204, 2001.

MEYLAN, C.; McMASTER, T.; CRONIN, J.; MOHAMMAD, N.I.; ROGERS, C.; DEKLERK, M. Single-leg lateral, horizontal, and vertical jump assessment: reliability, interrelationships, and ability to predict sprint and change-of-direction performance. Journal of Strength and Conditioning Research, Champaign, v.23, n.4, p.1140-7, 2009.

NORTON, K.; WHITTINGHAM, N.; CARTER, L.; KERR, D.; GORE, C.; MARFELL-JONES, M. Técnicas de mediçāo em antropometria. In: NORTON, K.; OLDS, T. Antropométrica. Porto Alegre: Artmed, 2005. p.41-87. 
NUZZO, J.L.; McBRIDE, J.M.; CORMIE, P.; McCAULLEY, G.O. Relationship between countermovement jump performance and multijoint isometric and dynamic tests of strength. Journal of Strength and Conditioning Research, Champaign, v.22, n.3, p.699-707, 2008.

RODACKI, A.L.; FOWLER, N.E. Intermuscular coordination during pendulum rebound exercises. Journal of Sports Sciences, London, v.19, n.6, p.411-25, 2001.

RODACKI, A.L.; FOWLER, N.E.; BENNETT, S.J. Multi-segment coordination: fatigue effects. Medicine and Science in Sports and Exercise, Madison, v.33, n.7, p.1157-67, 2001.

SMIRNIOTOU, A.; KATSIKAS, C.; PARADISIS, G.; ARGEITAKI, P.; ZACHAROGIANNIS, E.; TZIORTZIS, S. Strength-power parameters as predictors of sprinting performance. Journal of Sports Medicine and Physical Fitness, Turin, v.48, n.4, p.447-54, 2008. THORLUND, J.B.; AAGAARD, P.; MADSEN, K. Rapid muscle force capacity changes after soccer match play. International Journal of Sports Medicine, Stuttgart, v.30, n.4, p.273-8, 2009.

THORLUND, J.B.; MICHALSIK, L.B.; MADSEN, K.; AAGAARD, P. Acute fatigue-induced changes in muscle mechanical properties and neuromuscular activity in elite handball players following a handball match. Scandinavian Journal of Medicine \& Science in Sports, Copenhagen, v.18, n.4, p.462-72, 2008.

UGARKOVIC, D.; MATAVULJ, D.; KUKOLJ, M.; JARIC, S. Standard anthropometric, body composition, and strength variables as predictors of jumping performance in elite junior athletes. Journal of Strength and Conditioning Research, Champaign, v.16, n.2, p.227-30, 2002.

UGRINOWITSCH, C.; TRICOLI, V.; RODACKI, A.L.; BATISTA, M.; RICARD, M.D. Influence of training background on jumping height. Journal of Strength and Conditioning Research, Champaign, v.21, n.3, p.848-52, 2007.

WINTER, D.A. Kinematics. In: BIOMECHANICS and motor control of human movement. 3rd ed. New Jersey: John Wiley \& Sons, 2004. p.13-58.

YAMAUCHI, J.; ISHII, N. Relations between force-velocity characteristics of the knee-hip extension movement and vertical jump performance. Journal of Strength and Conditioning Research, Champaign, v.21, n.3, p.703-9, 2007.

ZIV, G.; LIDOR, R. Vertical jump in female and male basketball players-A review of observational and experimental studies. Journal of Science and Medicine in Sport, Belconnen, v.13, n.3, p.332-9, 2009.

\section{Agradecimentos}

Este estudo contou com o apoio da Coordenação de Aperfeiçoamento de Pessoal de Nível Superior.

Os autores agradecem ao Prof. João Nunes e toda a comissão técnica da Seleção Brasileira de Basquetebol, assim como, em especial as atletas da seleção, pela imensa colaboração e disposição para com essa investigação.

ENDEREÇO

Rodrigo Maciel Andrade Escola de Educação Física e Esporte - USP Av. Prof. Mello Moraes, 65 05508-030 - São Paulo - SP - BRASIL e-mail: rodmaciel@gmail.com
Recebido para publicação: 01/ 07/2011

1a. Revisão: 16/11/ 2011

2a. Revisão: 08/ 12/ 2011

3a. Revisão: 13/02/2012

Aceito: 24/02/2012 